\title{
QT dispersion in patients with chronic heart failure: $\beta$ blockers are associated with a reduction in QT dispersion
}

\author{
C E Bonnar, A P Davie, L Caruana, L Fenn, S A Ogston, J J V McMurray, A D Struthers
}

\begin{abstract}
Objective-To compare QT dispersion in patients with impaired left ventricular systolic function and in matched control patients with normal left ventricular systolic function.

Design-A retrospective, case-control study with controls matched 4:1 for age, sex, previous myocardial infarction, and diuretic and $\beta$ blocker treatment.

Setting-A regional cardiology centre and a university teaching hospital.

Patients-25 patients with impaired left ventricular systolic function and 100 patients with normal left ventricular systolic function.

Main outcome measures-QT and QTc dispersion measured by three methods: the difference between maximum and minimum QT and QTc intervals, the standard deviation of QT and QTc intervals, and the "lead adjusted" QT and QTc dispersion.

Results-All measures of QT/QTc dispersion were closely interrelated ( $r$ values 0.86 to 0.99 ; all $p<0.001)$. All measures of QT and QTc dispersion were significantly increased in the patients with impaired left ventricular systolic function $v$ controls $(\mathrm{p}<0.001)$ : 71.9 (6.5) (mean (SEM)) $v$ 46.9 (1.7) $\mathrm{ms}$ for QT dispersion, and 83.6 (7.6) $v 54.3(2.1) \mathrm{ms}^{-\frac{1}{2}}$ for QTc dispersion. All six dispersion parameters were reduced in patients taking $\beta$ blockers $(p<0.05)$, regardless of whether left ventricular function was normal or impaired-by 9.4 (4.6) $\mathrm{ms}$ for QT dispersion $(p<0.05)$ and by $13.8(6.5) \mathrm{ms}^{-\frac{1}{2}}$ for QTc dispersion $(p=0.01)$.

Conclusions-QT and QTe dispersion are increased in patients with systolic heart failure in comparison with matched controls, regardless of the method of measurement and independently of possible confounding factors. $\beta$ Blockers are associated with a reduction in both QT and QTc dispersion, raising the possibility that a reduction in dispersion of ventricular repolarisation may be an important antiarrhythmic mechanism of $\beta$ blockade. (Heart 1999;81:297-302)
\end{abstract}

Keywords: QT dispersion; heart failure; $\beta$ blockers; sudden death

Clinical and experimental electrophysiological studies have shown the importance of inhomo- geneous myocardial repolarisation in the genesis of ventricular arrhythmias. ${ }^{1-3}$ Increased dispersion of repolarisation provides a substrate for ventricular arrhythmias by generating areas of functional unidirectional block, thereby predisposing to reentry. ${ }^{3} \mathrm{QT}$ dispersion, defined as the difference between the longest and the shortest QT interval on the surface ECG, is a validated measure of dispersion of repolarisation. ${ }^{4} \mathrm{QT}$ dispersion predicts sudden death and ventricular arrhythmias in patients with chronic heart failure from coronary heart disease ${ }^{56}$ and in hypertrophic obstructive cardiomyopathy. ${ }^{7}$ QT dispersion also predicts cardiac death in patients with hypertension $^{8}$ and peripheral vascular disease. ${ }^{9}$ In patients with chronic heart failure from idiopathic dilated cardiomyopathy, QT dispersion has no predictive value for sudden death or ventricular arrhythmias ${ }^{10}{ }^{11}$ and there have been conflicting reports of its prognostic value in patients with acute myocardial infarction. ${ }^{12} 13$

There are few controlled studies of whether QT dispersion is abnormal in chronic heart failure. Two studies in patients with heart failure from coronary heart disease reported conflicting findings ${ }^{6}{ }^{14}-\mathrm{QT}$ dispersion was not increased in one ${ }^{14}$ but was increased in the other. ${ }^{6}$ In the latter study, however, $60 \%$ of patients had a previous myocardial infarct, a clinical event thought to increase QT dispersion even in the presence of preserved left ventricular systolic function. ${ }^{15}$ Two other studies in patients with chronic heart failure caused by idiopathic dilated cardiomyopathy showed increased QT dispersion. ${ }^{11}{ }^{16}$ However, both of these studies, like the previous two, used healthy controls, which is less than ideal because they are not matched for the possible effects of drugs, ${ }^{17}$ diuretic induced electrolyte abnormalities, ${ }^{18}{ }^{19}$ or sex imbalance. ${ }^{20}$

The aim of our study was therefore to compare the QT dispersion of a group of patients with impaired left ventricular systolic function with a large number of control patients who had normal systolic function but were matched for other confounding variables such as age, sex, diuretic and $\beta$ blocker treatment, and previous myocardial infarction. To perform such a matched study, we chose a unique population of relatively unselected, breathless, diuretic treated patients referred because of suspected heart failure for echocardiography. ${ }^{21}$ Some of these patients did and some did not have left ventricular systolic dysfunction. ${ }^{21}$ 
Methods

Patients with suspected heart failure were studied at the open access echocardiography service at the Western General Hospital, Edinburgh, which has been described previously. ${ }^{21}$ Briefly, current symptoms, past medical history, conventional risk factors, and current cardioactive drugs by class were recorded for each patient. All patients had ECG records made at the same paper speed and gain setting (25 $\mathrm{mm} / \mathrm{ms}$ and $10 \mathrm{~mm} / \mathrm{mV}$, respectively). Full cross sectional, $M$ mode, colour flow, and Doppler echocardiographic studies were then performed by an experienced technician and reported by a cardiologist. Left ventricular systolic function was quantified in terms of fractional shortening and was considered to be significantly impaired if it was less than $25 \%$ in the absence of valvar regurgitation. In nonechogenic subjects, a semiquantitative assessment of left ventricular systolic function was made, being either normal or impaired.

\section{PATIENT GROUPS}

From the first 534 patients referred to the open access service, 96 had significantly impaired left ventricular systolic function and 438 had preserved left ventricular systolic function. ${ }^{22}$ Patients were excluded from the analysis if they had any of the following: atrial fibrillation, atrial flutter, right or left bundle branch block (BBB), electrocardiographic left ventricular hypertrophy (ECG-LVH), or hypertension. QT dispersion is significantly increased in patients with hypertension, correlating with systolic blood pressure and left ventricular mass index, so we wished to avoid this possible confounding influence. ${ }^{23}$

Of the 96 patients with impaired left ventricular systolic function, 38 had one of the exclusion criteria (atrial fibrillation 6, BBB 14, ECG-LVH 12, hypertension 6) and 29 patients had two or more exclusion criteria. In the remaining 29 patients, QT dispersion could not be measured in four cases because of frequent ventricular ectopic beats (2) or poor quality ECG recordings (2), leaving 25 patients with impaired left ventricular systolic function.

Of the 438 patients with preserved left ventricular systolic function, 171 had one of the exclusion criteria (atrial fibrillation 18, atrial flutter 2, BBB 21, ECG-LVH 22, hypertension 108) and 65 had two or more exclusion criteria, leaving 202 patients. The 25 patients with impaired left ventricular systolic function were then matched as closely as possible for $\beta$ blocker treatment, diuretic treatment (loop, thiazide, or combination), sex, age, and previous myocardial infarction with 100 of the 202 patients with preserved left ventricular systolic function. We then compared QT and QTc dispersion in each of these groups.

\section{QT DISPERSION}

All ECGs were digitised by a single observer, blinded to the assigned group of each patient. ECGs were digitised manually with the aid of a digitising tablet connected to a personal computer. The QT interval was measured from the beginning of the QRS complex to the end of the $\mathrm{T}$ wave, defined as the return to T-P baseline. When $U$ waves were present, the QT interval was measured to the nadir of the curve between the $\mathrm{T}$ and $\mathrm{U}$ waves. QT intervals were measured in all leads if possible. The QT interval could be measured in at least seven leads in all patients in the study. For each lead, two or three consecutive cycles were measured and the arithmetic mean of the QT interval for that lead was used in all future calculations for QT dispersion.

We measured QT dispersion in three different ways. First, QT dispersion was defined as the difference between the maximum and minimum measured QT intervals across the 12 lead ECG. This is entirely dependent on the values observed in just two of the 12 leads and is markedly influenced by the number of leads in which the QT interval can be properly measured. ${ }^{24}$ Second, we measured the standard deviation of all QT intervals in each ECG (SD-QT interval). This incorporates information from all measured leads, not just maximum and minimum values; of all the formulas used for QT dispersion it is the least affected by using different numbers of leads in different patients. ${ }^{24}$ Third, "adjusted" QT dispersion is QT dispersion divided by the square root of the number of leads in which the QT interval was measured, a formula proposed by Day et al to compensate for using different numbers of leads in different patients. ${ }^{25}$ All three formulas were then expressed as both "uncorrected" QT dispersion and "rate corrected" QTc dispersion, using Bazett's formula. ${ }^{26}$

\section{STATISTICAL ANALYSIS}

Statistical tests were performed using a dedicated software package (SPSS version 7.5 for Windows-NT); $\chi^{2}$ analysis was used to compare differences in the baseline characteristics in the groups, and simple linear regression to test for associations between variables. For between group comparisons, one way analysis of variance (ANOVA) and multiple regression analysis were used. A $p$ value $<0.05$ was considered to be significant. Values throughout the text and in the tables are expressed as mean (SEM).

\section{Results}

\section{PATIENT CHARACTERISTICS}

Table 1 outlines the baseline characteristics for each group of patients and shows that the groups were well balanced. We were unable to match precisely for previous myocardial infarction or diabetes, both of which were more common in the chronic heart failure group $(p<0.05)$. These imbalances were taken into account in the multiple regression analysis, which adjusted for any possible increase in QT/QTc dispersion indices in the chronic heart failure group caused by these differences. In addition, seven patients with impaired left ventricular systolic function were taking calcium antagonists, seven were taking long acting nitrates, four were taking angiotensin converting enzyme (ACE) inhibitors, and three were taking digoxin. Of the 100 patients with preserved left ventricular systolic function, 15 
Table 1 QT and QTc dispersion in patients with impaired left ventricular systolic function and matched controls with preserved left ventricular systolic function

\begin{tabular}{|c|c|c|c|}
\hline & \multicolumn{2}{|c|}{ Left ventricular function } & \multirow[b]{2}{*}{$p$ Value } \\
\hline & Impaired & Preserved & \\
\hline Group & 1 & 2 & \\
\hline Number of patients & 25 & 100 & \\
\hline Age (years) & $69.2(2.1)$ & $67.7(1.5)$ & †NS \\
\hline Male $(\%(n))$ & $48 \%(12 / 25)$ & $49 \%(49 / 100)$ & NS \\
\hline Diuretics (\% (n)) & $68 \%(17 / 25)$ & $63 \%(63 / 100)$ & NS \\
\hline$\beta$ Blockers $(\%(n))$ & $16 \%(4 / 25)$ & $13 \%(13 / 100)$ & NS \\
\hline Diabetes mellitus (type I or II) (\% (n)) & $24 \%(6 / 25)$ & $3 \%(3 / 100)$ & $<0.01$ \\
\hline Previous history of MI ((\% (n)) & $52 \%(13 / 25)$ & $23 \%(23 / 100)$ & $<0.05$ \\
\hline QT dispersion (ms) & $71.9(6.5)$ & $46.9(1.7)$ & $\star<0.001$ \\
\hline QTc dispersion $\left(\mathrm{ms}^{-\frac{1}{2}}\right)$ & $83.6(7.6)$ & $54.3(2.1)$ & $\star<0.001$ \\
\hline SD-QT interval (ms) & $24.4(2.6)$ & $14.8(0.5)$ & $\star<0.001$ \\
\hline SD-QTc interval $\left(\mathrm{ms}^{-\frac{1}{2}}\right)$ & $28.1(3.0)$ & $17.3(0.7)$ & $\star<0.001$ \\
\hline Ad.QT dispersion (ms) & $22.9(2.1)$ & $14.7(0.5)$ & $\star<0.001$ \\
\hline Ad.QTc dispersion $\left(\mathrm{ms}^{-\frac{1}{2}}\right)$ & $26.7(2.4)$ & $17.0(0.7)$ & $\star<0.001$ \\
\hline
\end{tabular}

Values are mean (SEM) unless otherwise stated; $\chi^{2}$ analysis except: $\dagger$ Student's $t$ test; ${ }^{\star}$ analysis of variance.

Ad.QT dispersion, "lead adjusted" QT dispersion; Ad.QTc dispersion, "lead adjusted" QTc dispersion; MI, myocardial infarction; QTc dispersion, QT dispersion corrected for heart rate using Bazett's formula; SD-QT interval, standard deviation of the QT interval; SD-QTc interval, standard deviation of the rate corrected QT interval.

Table 2 QT and QTc dispersion in patients not taking and taking chronic $\beta$ blocker treatment

\begin{tabular}{llcl}
\hline & \multicolumn{2}{l}{ Chronic $\beta$ blocker treatment } & \\
\cline { 2 - 3 } & No & Yes & p Value \\
\hline Number of patients & 108 & 17 & \\
QT dispersion (ms) & $53.2(1.9)$ & $43.8(4.9)$ & $<0.05$ \\
QTc dispersion (ms & \\
SD- QT interval $\left(\mathrm{ms}^{-\frac{1}{2}}\right)$ & $62.0(2.4)$ & $48.2(6.1)$ & 0.01 \\
SD-QTc interval $\left(\mathrm{ms}^{-\frac{1}{2}}\right)$ & $17.1(0.7)$ & $14.2(1.7)$ & $<0.05$ \\
Ad.QT dispersion $\left(\mathrm{ms}^{-\frac{1}{2}}\right.$ & $20.1(0.9)$ & $15.6(2.2)$ & $<0.05$ \\
Ad.QTc dispersion (ms & $16.8(0.6)$ & $13.7(1.5)$ & $<0.05$ \\
& $19.6(0.8)$ & $15.0(1.9)$ & $<0.01$ \\
\hline
\end{tabular}

*Analysis of variance adjusted for the factors and covariates in the multiple regression. Ad. QT dispersion, "lead adjusted" QT dispersion; Ad. QTc dispersion, "lead adjusted" QTc dispersion; SD-QT interval, standard deviation of QT interval; SD-QTc interval, standard deviation of the rate corrected QT interval.

were taking calcium antagonists, 13 were taking long acting nitrates, six were taking ACE inhibitors, and one was taking digoxin.

QT DISPERSION

Our results showed that QT and QTc dispersion were significantly increased in patients with chronic heart failure, regardless of the method used to measure them and independently of possible confounding factors (table 1).

MULTIPLE REGRESSION ANALYSIS

Eleven variables were used in the multiple regression analysis (patient group, age, sex, diabetes mellitus, previous myocardial infarction, diuretics, $\beta$ blockers, ACE inhibitors, nitrates, calcium antagonists, and digoxin). Of these, only patient group (presence or absence of impaired left ventricular systolic function) and $\beta$ blocker treatment were significantly related to all six QT/QTc dispersion indices. Previous myocardial infarction increased QT dispersion $(\mathrm{p}<0.05)$ and SD-QT interval $(\mathrm{p}<0.05)$ but this did not reach statistical significance for the four other QT/QTc dispersion indices $(p=0.08$ to 0.17$)$. Diabetes had no significant effect on any of the QT/QTc dispersion indices, and entering diabetes into the multiple regression model did not influence the results in any way. All three QT dispersion indices were significantly increased by digoxin treatment: QT dispersion was increased by 30.1 (10.2) ms ( $\mathrm{p}=0.02)$, SD-QT interval by
11.4 (3.6) $\mathrm{ms}(\mathrm{p}<0.01)$, and adjusted QT dispersion by $9.7(3.2) \mathrm{ms}(\mathrm{p}=0.01)$. Of the QTc dispersion indices, only adjusted QTc dispersion was increased by digoxin treatment, by $9.3(4.0) \mathrm{ms}^{-\frac{1}{2}}(\mathrm{p}=0.05)$. Treatment with ACE inhibitors reduced SD-QTc interval by 8.7 (2.9) $\mathrm{ms}^{-\frac{1}{2}}(\mathrm{p}=0.04)$ but the other QT/QTc dispersion indices were unaffected.

\section{Patient group}

The presence or absence of impaired left ventricular systolic function was the most important factor that influenced all six QT/ QTc dispersion parameters $(\mathrm{p}<0.001$, for all).

\section{$\beta$ Blocker treatment}

All six measures of QT/QTc dispersion were significantly lower in patients taking $\beta$ blocker treatment compared with those not taking it $(\mathrm{p}<0.05)$ (table 2). QT dispersion was lower by $9.4(4.6) \mathrm{ms}(\mathrm{p}<0.05)$ and QTc dispersion by $13.8(6.5) \mathrm{ms}^{-\frac{1}{2}}(\mathrm{p}=0.01)$ in patients taking $\beta$ blockers (table 2). To assess whether this effect was present in patients with impaired and preserved left ventricular function, we added an extra variable to the multiple regression model (interaction of patient group with $\beta$ blocker). This was not significant, confirming that the effect observed was independent of left ventricular function.

In the group taking $\beta$ blockers $(n=17)$, one patient with a history of ventricular aneurysm which had been surgically repaired had a QT dispersion of $94.7 \mathrm{~ms}$ and a QTc dispersion of $103.8 \mathrm{~ms}^{-\frac{1}{2}}$. In the remaining 16 patients, QT dispersion was less than $63.3 \mathrm{~ms}$ and QTc dispersion less than $67.3 \mathrm{~ms}^{-\frac{1}{2}}$. In contrast, patients not taking $\beta$ blockers $(n=108)$ had QT dispersion values as high as $134.7 \mathrm{~ms}$ and QTc dispersion values as high as $162.0 \mathrm{~ms}^{-\frac{1}{2}}$.

\section{Discussion}

We have shown for the first time that QT dispersion is increased in patients with chronic heart failure compared with well matched controls. Previous studies of QT dispersion in patients with chronic heart failure have tended to compare healthy controls with patients with a past history of myocardial infarction and who are receiving pharmacological treatment. ${ }^{6} 1114$ It has therefore previously been impossible to determine whether abnormal QT dispersion in these patients was caused by left ventricular dysfunction, previous infarction, ${ }^{15}$ or drug treatment. ${ }^{17}$ Our findings show that QT dispersion is increased in patients with systolic heart failure compared with appropriately matched breathless patients without systolic heart failure. Our finding that four of the six measures of QT/QTc dispersion were not significantly increased in patients with a previous myocardial infarct suggests that it is the left ventricular dysfunction per se rather than the previous infarction that leads to abnormal QT dispersion. Furthermore, we found that digoxin treatment increased four of the six QT/QTc dispersion indices and that chronic diuretic treatment was not associated with any effect on QT dispersion. 
One interesting finding that emerged from this analysis was that chronic $\beta$ blocker treatment is associated with reduced QT and QTc dispersion, regardless of whether left ventricular function was preserved or impaired. This was observed in 16 of the 17 patients in the study who were taking $\beta$ blockers (one patient with a surgically repaired ventricular aneurysm had increased QT dispersion despite $\beta$ blockade, presumably as a result of distorted ventricular anatomy). It is unlikely that the effect we observed is an anti-ischaemic one, since nitrates and calcium antagonists (which were used by more of the patients then were $\beta$ blockers) were not associated with any index of QT or QTc dispersion.

Little is known about the aetiology of increased QT dispersion in patients with chronic heart failure, but sympathetic tone, excitation-contraction coupling, and myocardial fibrosis may all be important. Sympathetic tone is greatly increased in patients with chronic heart failure compared to controls, as shown by studies of plasma and urinary catecholamines, noradrenaline spillover techniques, and heart rate variability, ${ }^{27}$ and this occurs even in patients with mild or asymptomatic chronic heart failure. Angiotensin II in particular exerts a marked stimulatory effect on central sympathetic modulation, noradrenaline secretion from sympathetic nerve terminals, and adrenergic receptor responsiveness. ${ }^{28}$ Chronic ACE inhibitor treatment in patients with chronic heart failure is associated with a reduction in plasma noradrenaline and muscle sympathetic nerve traffic ${ }^{27}$ (implying sympathetic deactivation), and we have previously shown that six weeks of treatment with enalapril in patients with mild asymptomatic chronic heart failure reduces QT dispersion. ${ }^{29}$ This, together with our present finding that QT dispersion is lower in patients taking chronic $\beta$ blocker treatment, suggests that sympathetic activation may be an important cause of increased QT dispersion in patients with chronic heart failure (the fact that ACE inhibitors did not reduce QT dispersion in this study was probably due to the small numbers of patients taking them).

Three other pieces of evidence support this theory. First, we have recently shown that QT dispersion significantly increases at dawn in patients with chronic heart failure, in parallel with the dawn surge in sympathetic activity. ${ }^{30}$ Second, we have also recently shown that QT dispersion correlates significantly with the low frequency component of heart rate variability (expressed in normalised units), which is an accepted measure of sympathetic nervous system activity. ${ }^{31}$ Third, intravenous and intracoronary salbutamol increase QTc dispersion in patients with coronary artery disease. ${ }^{32}$ Finally, it is worth pointing out that in one of the earliest studies investigating dispersion of ventricular repolarisation, $\mathrm{Han}$ and Moe showed that sympathetic activation increases dispersion of refractoriness and reduces ventricular fibrillation threshold. ${ }^{1}$

There are other possible reasons why QT dispersion is increased in patients with chronic heart failure. All types of heart failure, regardless of aetiology, are associated with changes in left ventricular size, function, or pressure, ${ }^{33}$ each of which may exert important electrophysiological effects (excitation-contraction coupling), including an increase in the dispersion of ventricular repolarisation. ${ }^{34}$ Previous studies, however, have reported weak correlations between QT dispersion and indices of left ventricular size or function in patients with chronic heart failure. ${ }^{14}$ Myocardial fibrosis has also been proposed as a cause of increased QT dispersion in patients with chronic heart failure, mainly because of the finding that patients with diffuse coronary artery disease have considerably greater QT dispersion than patients with one, two, or three vessel disease. ${ }^{9}$

In patients with idiopathic long QT syndrome, Priori et al showed that those responding to $\beta$ blocker treatment had significantly reduced QT dispersion compared with non-responders. ${ }^{35}$ As in our study, this suggested but did not prove that $\beta$ blockers reduce QT dispersion. It is also worth pointing out that complete removal of $\beta$ adrenergic activity by left cardiac sympathetic denervation in the study by Priori et al significantly reduced QT dispersion as well. ${ }^{35}$ Sotalol reduces QTc dispersion in patients after acute myocardial infarction but this effect has been attributed to its class III and not to its class II properties. ${ }^{25}$

Our findings suggest that $\beta$ blockers may independently reduce both QT and QTc dispersion, which could be of considerable significance in explaining their ability to prevent ventricular arrhythmias and sudden cardiac death after acute myocardial infarction. ${ }^{36} \mathrm{Al}-$ though many mechanisms of action have been proposed to explain the beneficial effects of $\beta$ blockers, the precise antiarrhythmic mechanism has not yet been identified. ${ }^{36}$ The traditional view has arisen that the effects of $\beta$ blockers on cardiac repolarisation are not of crucial significance in mediating their antiarrhythmic effects, ${ }^{37}$ although few studies have specifically examined whether chronic $\beta$ blockade actually influences ventricular repolarisation. The available studies do suggest an effect, since monophasic action potential duration, ventricular effective refractory periods, and QT intervals all increase with chronic $\beta$ blocker treatment. ${ }^{38-40}$ Fewer studies still have examined whether chronic $\beta$ blockade influences the dispersion of ventricular repolarisation, presumably because this used to be so difficult to measure by invasive endocardial catheter mapping or cumbersome body surface mapping. With the advent of QT dispersion, this has become much easier, albeit more indirect. Our study, and that of Priori et al, ${ }^{35}$ suggests that an important additional antiarrhythmic property of chronic $\beta$ blockade may be the ability to reduce increased dispersion of ventricular repolarisation, an important precursor of re-entry.

As in the majority of studies of QT dispersion all QT intervals in our study were measured manually, with the aid of a digitiser. There is evidence that manual measurement is superior to automatic measurement of QT dis- 
persion, which usually needs some form of manual editing ${ }^{41}$ and gives different results. ${ }^{42}$ The fact that our controls were not normal, healthy patients - in the sense that the majority had symptoms leading to a suspicion of heart failure - is not a limitation of our study but an overall advantage, since our control group was ideal in that they could be matched to our patients for nearly all confounding variables.

\section{LIMITATIONS}

Our study was a retrospective observational study and as such has several well known limitations. The patients in the study were generally not taking ACE inhibitors and so are representative of chronic heart failure patients as they present, rather than when they are optimally treated. In addition, we had no information on electrolytes in any of our patients but by controlling for diuretic treatment, we hoped to control for hypokalaemia and hypomagnesaemia, both of which have been shown to increase QT dispersion. ${ }^{18}{ }^{19}$ Also, we had no information on the final diagnoses of patients referred with suspected heart failure who were subsequently shown to have normal left ventricular systolic function. Likely causes of breathlessness in these patients - such as chronic obstructive pulmonary disease (COPD) - may have influenced QT dispersion. ${ }^{43}$ However, QT dispersion in patients with COPD is increased compared with controls, ${ }^{43}$ and this would have been expected to reduce and not increase the difference we observed between the two study groups.

\section{CONCLUSION}

In summary, we found that QT dispersion measured by several methods is increased in patients with systolic heart failure compared with appropriately matched breathless patients without systolic heart failure. Our findings suggest that it is the presence of left ventricular systolic dysfunction per se and not a previous myocardial infarct that increases QT dispersion in patients with chronic heart failure. Our findings also suggest that $\beta$ blockers are capable of independently reducing both QT and QTc dispersion, raising the possibility that an important and previously unrecognised antiarrhythmic property of $\beta$ blockers may be the ability to reduce dispersion of ventricular repolarisation. This needs to be confirmed in a prospective, randomised, placebo controlled study.

1 Han J, Moe GK. Nonuniform recovery of excitability in ventricular muscle. Circ Res 1964;14:44-60.

2 Vassallo JA, Cassidy DM, Kindwall KE, et al. Non uniform recovery of excitability in the left ventricle. Circulation 1988;78:1365-72.

3 Kuo CS, Munakata K, Reddy CP, et al. Characteristics and possible mechanisms of ventricular arrhythmia dependent on the dispersion of action potential duration. Circulation 1983;67:1356-67.

4 Zabel M, Portnoy J, Franz M. Electrocardiographic indexes of dispersion of ventricular repolarization: an isolated heart validation study. F Am Coll Cardiol 1995;25:746-52.

5 Barr CS, Naas A, Freeman M, et al. QT dispersion and sudden unexpected death in chronic heart failure. Lancet 1994;343:327-9.

6 Fu GS, Meissner A, Simon R. Repolarization dispersion and sudden cardiac death in patients with impaired left sudden cardiac death in patients with imp
ventricular function. Eur Heart $\mathcal{f}$ 1997:18:281-9.

7 Buja G, Miorelli M, Turrini P, et al. Comparison of QT dispersion in hypertrophic cardiomyopathy between patients with and without ventricular arrhythmias and sudden death. Am 7 Cardiol 1993;72:973-6.

8 Galinier M, Balanescu S, Fourcade J, et al. Prospective study of prognostic value of arrhythmogenic markers in systemic hypertension [abstract]. 7 Am Coll Cardiol 1997;29:347A.

9 Darbar D, Luck J, Davidson N, et al. Sensitivity and specificity of QTc dispersion for identification of risk of cardiac death in patients with peripheral vascular disease. $B M \mathcal{F}$ 1996;312:874-8.

10 Strunk-Mueller C, Gietzen F, Kuhn H. QTc dispersion in dilated cardiomyopathy - a new method for stratifying the risk of sudden cardiac death? [abstract] Eur Heart $\mathcal{F}$ 1996; 94:I 276.

11 Grimm W, Steder U, Menz U, et al. QT dispersion and arrhythmic events in idiopathic dilated cardiomyopathy. Am $\mathcal{F}$ Cardiol 1996;78:458-61.

12 Potratz J, Djonlagic H, Brandes A, et al. Prognostic significance of the QT-dispersion in patients with acute myocardial infarction [abstract]. Circulation 1993:88:307.

13 Glancy JM, Garratt CJ, Woods KL, et al. QT dispersion and mortality after myocardial infarction. Lancet 1995;345: 945-8.

14 Davey PP, Bateman J, Mulligan IP, et al. QT-interval dispersion in chronic heart failure and left ventricular hypertrophy: relation to autonomic nervous system and Holter tape abnormalities. Br Heart f 1994;71:268-73.

5 Perkiomaki JS, Koistinen MJ, Yli-Mayry S, et al. Dispersion of QT-interval in patients with and without susceptibility to ventricular tachyarrhythmias after previous myocardial infarction. F Am Coll Cardiol 1995;26:174-9.

16 Zaidi M, Robert A, Fesler R, et al. Dispersion of ventricular repolarization in dilated cardiomyopathy. Eur Heart $\mathcal{f}$ 1997;18:1129-34.

17 Laakso M, Aberg A, Savola J, et al. Diseases and drugs causing prolongation of the QT-interval. Am $\mathcal{F}$ Cardiol 1987;59: $862-5$.

18 Yelamanchi V, Ranade V, Cao W, et al. Influence of electrolyte abnormality on interlead variability of ventricular repolarization times in 12-lead ECG [abstract]. $\mathcal{F}$ Am Coll Cardiol 1997;29:148A

19 Haigney MC, Berger R, Calkins H, et al. QT dispersion correlates with tissue magnesium concentration in humans [abstract]. Circulation 1994;90(suppl I):248.

20 Challapalli S, Lingamneni R, Ehlert F, et al. Gender differences in QT-dispersion [abstract]. $\mathcal{F}$ Am Coll Cardiol 1997; 29:148A.

21 Francis CM, Caruana L, Kearney P, et al. Open access echocardiography in management of heart failure in the community. BMF 1995;310:634-6.

22 Davie AP, Francis CM, Love MP, et al. Value of electrocardiogram in identifying heart failure due to left ventricular diogram in identifying heart failure due to
systolic dysfunction. $B M \mathcal{F} 1996 ; 312: 222-4$.

23 Mayet J, Shahi M, McGrath C, et al. Left ventricular hypertrophy and QT dispersion in hypertension. Hypertension 1996;28:791-6

24 Hnatkova K, Malik M, Kautzner J, et al. Adjustment of QT dispersion assessed from 12 lead electrocardiograms for different numbers of analysed electrocardiographic leads: comparison of stability of different methods. Br Heart $\mathcal{F}$ 1994;72:390-6.

25 Day CP, McComb JM, Matthews J, et al. Reduction in QT dispersion by sotalol following myocardial infarction. Eur Heart f 1991;12:423-7.

26 Bazett HC. An analysis of the time relations of electrocardiograms. Heart 1920:7:353.

27 Grassi G, Cattaneo BM, Mancia G. Sympathetic nervous system. In: Poole-Wilson PA, ed. Heart failure. New York: Churchill Livingstone, 1997:199-214.

28 Mancia G, Giannattasio C, Grassi G, et al. Reflex control of circulation and ACE inhibition in man [abstract]. F Hypercirculation and ACE inhibit
tens $1988 ; 6$ (suppl 3):S45.

29 Barr CS, Naas AA, Fenwick M, et al. Enalapril reduces QT dispersion in patients with mild congestive heart failure secondary to coronary artery disease. Am f Cardiol 1997;79:328-33.

30 Bonnar CE, Gillespie ND, MacFadyen RJ, et al. QT dispersion is significantly increased between 6am and $8 \mathrm{am}$ in heart failure patients - a possible role in sudden death? [abstract] f Am Coll Cardiol 1997;29(suppl A):510A.

31 Bonnar CE, MacFadyen RJ, Robson JM, et al. QT dispersion is related to autonomic tone in patients with stable chronic heart failure [abstract]. Eur Heart f 1997;18: 200.

32 Lowe MD, Ludman PF, Rowland E, et al. $\beta$ Adrenoceptor stimulation increases dispersion of cardiac repolarisation [abstract]. Clin Sci 1997;93:1p.

33 Pye MP, Cobbe SM. Mechanisms of ventricular arrhythmia in cardiac failure and hypertrophy. Cardiovasc Res 1992;26: $740-50$

34 Dean JW, Lab MJ. Arrhythmia in heart failure. Role of mechanically induced changes in electrophysiology. Lancet 1989;ii: 1309-12

35 Priori SG, Napolitano C, Diehl L, et al. Dispersion of the QT-interval-a marker of therapeutic efficacy in the idiopathic long QT-syndrome. Circulation 1994;89: 1681-9.

36 Frishman WH, Lazar EJ. Reduction of mortality, sudden death and non-fatal reinfarction with beta-adrenergic blockers in survivors of acute myocardial infarction: a of beta-adrenergic blockade. Am f Cardiol 1990;66:66$70 \mathrm{G}$.

37 Singh BN, Courtney KR. The classification of antiarrhythmic mechanisms of drug action: experimental and clinical 
considerations. In: Zipes DP, ed. Cardiac electrophysiology: from cell to bedside. Philadelphia: WB Saunders, 1990:882 97.

38 Edvardsson N, Olsson SB. Effects of acute and chronic beta-receptor blockade on ventricular repolarization in man. Br Heart f 1981;45:628-36.

39 Kentsch M, Kunze KP, Kuck KH. Sotalol and metoprolol in ventricular tachycardia: preliminary data of a double-blind prospective study [abstract]. F Am Coll Cardiol 1988; 11(suppl A):227A.

40 Raine AEG, Vaughan Williams EM. Adaptation to prolonged $\beta$-blockade of rabbit atrial, Purkinje and ventricular potentials and of papillary muscle contraction. Circ Res 1981;48:804-12.

41 Glancy JM, Weston PJ, Bhullar HK, et al. Reproducibility and automatic measurement of QT dispersion. Eur Heart $\mathcal{F}$ 1996;17:1035-9.

42 Murray A, McLaughlin NB, Campbell RWF. Measuring QT dispersion: man versus machine. Heart 1997;77:53942 .

43 Sarubbi B, Esposito V, Ducceschi V, et al. Effect of blood gas derangement on QTc dispersion in severe chronic obstructive pulmonary disease: evidence of an electropathy? Int $\mathcal{F}$ Cardiol 1997;58:287-92.

\section{IMAGES IN CARDIOLOGY}

\section{Biatrial thrombosis in cardiac amyloidosis}

A 60 year old woman was admitted to hospital because of congestive heart failure. Primary AL amyloidosis with extensive infiltration of the heart was diagnosed. The ECG showed sinus rhythm and low QRS voltage.

Transthoracic echocardiography typically documented a diffuse thickening of atrial and ventricular walls, preserved systolic ventricular function, and dilatation of the atria. In the body of left atrium a small thrombus was visible.

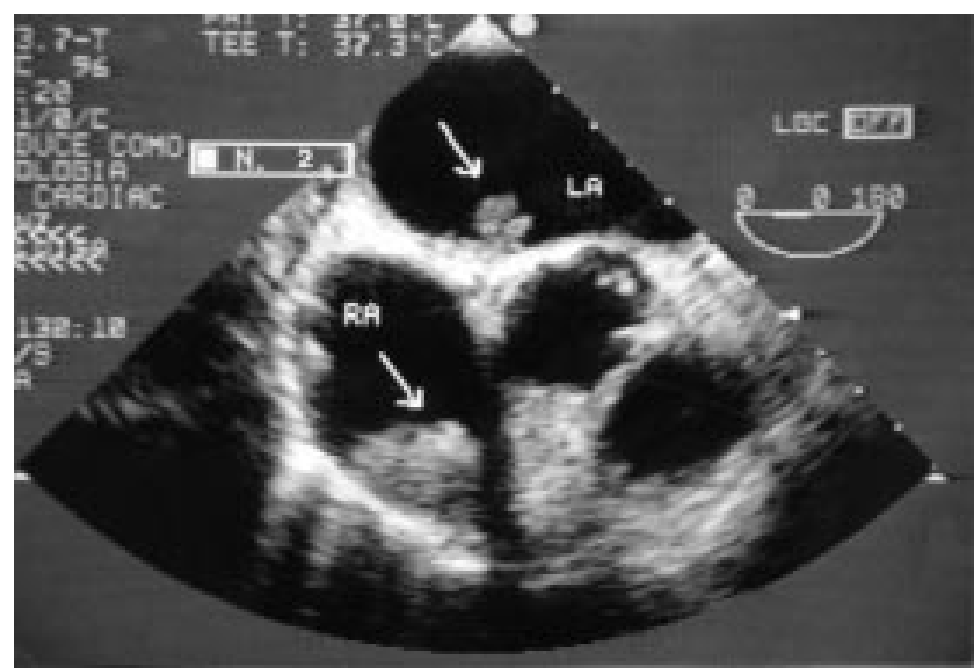

Transoesophageal echocardiography confirmed the presence of a roundish $1 \times 1 \mathrm{~cm}$ thrombus in the corner between interatrial septum and aorta. Moreover a $2 \times 3 \mathrm{~cm}$ non-mobile thrombus was detected in the right atrial appendage (figure). The left atrial appendage emptying velocity was much reduced.

Ten days after starting anticoagulation (intravenous heparin then warfarin), repeat transoesophageal echocardiography showed the resolution of the left atrial thrombus and a slight size reduction of the right atrial appendage thrombus. A few days later the patient had syncope, followed by shock and death. Necropsy revealed a massive pulmonary embolism; the right atrial appendage thrombus was still present.

In advanced forms of cardiac amyloidosis, an impairment of atrial emptying predisposes to atrial thrombosis, even in sinus rhythm. The atrial dysfunction has been ascribed to the combination of amyloid infiltration of atrial walls and an increase of atrial afterload owing to restrictive haemodynamics. Furthermore, clotting factors might play a role in thrombogenesis of primary systemic amyloidosis.

M SANTARONE G CORRADO

L M TAGLIAGAMBE 